\title{
Computing Zagreb and Randić Indices of PEG-Cored Dendrimers Used for Drug and Gene Delivery
}

\author{
Thayamathy Pio Jude, ${ }^{1}$ Elango Panchadcharam $\mathbb{D}^{1},{ }^{1}$ and Koneswaran Masilamani ${ }^{2}$ \\ ${ }^{1}$ Department of Mathematics, Faculty of Science, Eastern University Sri Lanka, Vantharumoolai, Chenkalady, \\ Batticaloa 30350, Sri Lanka \\ ${ }^{2}$ Department of Chemistry, Faculty of Science, Eastern University Sri Lanka, Vantharumoolai, Chenkalady, \\ Batticaloa 30350, Sri Lanka \\ Correspondence should be addressed to Elango Panchadcharam; elangop@esn.ac.lk
}

Received 3 August 2020; Revised 14 September 2020; Accepted 24 September 2020; Published 15 October 2020

Academic Editor: Maria N. D. S. Cordeiro

Copyright (C) 2020 Thayamathy Pio Jude et al. This is an open access article distributed under the Creative Commons Attribution License, which permits unrestricted use, distribution, and reproduction in any medium, provided the original work is properly cited.

Zagreb and Randić indices are the most commonly used degree-based topological indices in the study of drug design and development. In molecular topology, $M$-polynomials are also used to calculate the degree-based topological indices of chemical structures. In this paper, we derive the $M$-polynomials for the PEG-cored PAMAM, carbosilane, and poly (lysine) dendrimers and calculate their first, second, and second modified Zagreb indices and the Randić index.

\section{Introduction}

A topological index is a numerical quantity, which is derived mathematically in a direct and unambiguous manner from the structural graph of a molecule. They are calculated from the heavy atom graphical depiction of the molecule. The most commonly used topological indices in the field of drug design and development are the Wiener index, Kosoya index or $Z$ index, Zagreb index, Randić index, and Balaban $J$ index. Among these indices, the Wiener index, Kosoya index or $Z$ index, and Balaban $J$ indices are nondegree -based topological indices, while Zagreb and Randić indices are the degree-based topological indices. The degree-based topological indices are the most studied type of topological indices, which play an important role in the field of molecular graph theory or molecular topology. Topological indices are mostly used in the drug design and development process, and some of their roles are reported by researchers [1-5]. Recent studies on Zagreb and Randić indices are reported by various scientists [6-9]. Nowadays, many researchers are focused on dendrimers to study their various topological indices [10-12].

Dendrimers are manmade, nanoscale compounds with unique properties that make them useful in many applications, especially in the field of medical applications. These dendrimers are monodisperse molecule with a regular and highly branched three-dimensional architecture. The globular structure and excellent flexibility of dendrimers make them as a drug and gene delivery system [13-15].

In this work, we considered three types of PEG-cored dendrimers such as PEG-cored PAMAM, PEG-cored carbosilane, and PEG-cored triazine, and these dendrimers are used as promising candidates for drug and gene delivery with high drug encapsulation, sustained release behaviour, and excellent gene transfection efficiency. In this paper, we computed Zagreb index and Randić index for the abovementioned PEG-cored dendrimers, and these results could be used in determining properties of these compounds [16].

A molecular graph in the molecular graph theory or molecular topology is a simple graph which has neither loops nor multiple edges. In the molecular graph theory, the atoms and the chemical bonds between atoms are represented by vertices and edges, respectively. A graph $G=G(V, E)$ consists of a nonempty set of vertices $V=V(G)$ and the set of connected edges (if there exists a connection between any pair of vertices) $E=E(G)$. In a connected graph, the degree of $v$ is denoted by $d_{v}(G)$ or $d_{v}$ and is defined to be the number of vertices which are 
connected to that vertex by the edges. The concept of degree is closely related to the concept of valence bond in chemistry. The distance between two vertices $u$ and $v$ is denoted by $d(u, v)$ or $d_{G}(u, v)$ and is defined to be the length of shortest path between these vertices.

The Wiener index is the first and the most studied distance-based topological index in molecular topology. This was introduced by a chemist, Wiener [17] in 1947, to demonstrate the correlations between physicochemical properties of organic compounds and the topological structure of their molecular graphs. The Wiener index is defined as

$$
W(G)=\sum_{\{u, v\} \subseteq V(G)} d_{G}(u, v) .
$$

This is calculated as the sum of distances between all the carbon atoms in the molecules, in terms of carboncarbon bonds. Wiener named this index as the path number. The degree-based topological indices are the second type of topological indices, which are the most studied type of topological indices in molecular topology. One of the oldest degree-based topological indices is the well-known Zagreb index, which was introduced by Gutman and Trinajstić [18] in 1972 during the analysis of the structure-dependency of total $\pi$-electron energy. The name Zagreb is the place where both of them worked in an institute as members of the theoretical chemistry group. The first Zagreb index $M_{1}(G)$ and the second Zagreb index $M_{2}(G)$ s are defined as

$$
\begin{aligned}
& M_{1}(G)=\sum_{v \in V(G)}\left(d_{v}\right)^{2}=\sum_{u v \in E(G)}\left(d_{u}+d_{v}\right), \\
& M_{2}(G)=\sum_{u v \in E(G)} d_{u} d_{v} .
\end{aligned}
$$

The second modified Zagreb index [19] is defined as

$$
{ }^{m} M_{2} M_{2}(G)=\sum_{u v \in E(G)} \frac{1}{d_{u} d_{v}} .
$$

Another oldest degree-based topological index called as the Randić index was introduced by Randić [20] in 1974 and defined as

$$
R(G)=\sum_{u v \in E(G)} \frac{1}{\sqrt{d_{u} d_{v}}} .
$$

Randić named this index as the branching index, and soon, it was renamed as connectivity index. The Randić index is the most studied and most often applied degreebased topological index in the field of drug design. This index was globalized and called as the generalized Randić index [21]. The generalized Randić index is defined as

$$
R_{\alpha}(G)=\sum_{u v \in E(G)}\left(d_{u} d_{v}\right)^{\alpha},
$$

where $\alpha$ is an arbitrary real number. The Randić index could be obtained from this formula by substituting $\alpha=-1 / 2$.

\section{Materials and Methods}

Many graph polynomials play a prominent role to study the structural properties of the molecules. Among them, the Hosoya polynomial (also known as the Wiener polynomial) plays a vital role to determine the distance-based topological indices. Among the graph polynomials, the $M$-polynomial, which was introduced in 2015 by Deutsch and Klavzar [22], plays another important role to determine many degreebased topological indices.

For a graph $G=G(V, E)$, the $M$-polynomial is defined as

$$
M(G ; x, y)=\sum_{i \leq j} m_{i j}(G) x^{i} y^{j},
$$

where $m_{i j}(G), i, j \geq 1$ is the number of edges $e=u v$ of $G$ such that $\left\{d_{u}, d_{v}\right\}=\{i, j\}$, and $u, v \in V(G)$. The derived formulas to compute Zagreb and Randić indices from the $M$ polynomial are given in Table 1.

Here, $\quad D_{x}=x \partial f(x, y) / \partial x, D_{y}=y \partial f(x, y)$ $/ \partial y, S_{x}=\int_{0}^{x} f(t, y) / t \mathrm{~d} t$, and $S_{y}=\int_{0}^{y} f(x, t) / t \mathrm{~d} t$.

In this paper, we considered the three dendrimers, PEGcored PAMAM dendrimer (Figure 1), PEG-cored carbosilane dendrimer (Figure 2), and PEG-cored triazine dendrimer (Figure 3), which are used for drug and gene delivery. We first calculate the $M$-polynomial and then find the first, second, and the second modified Zagreb indices and the Randić index for each of the dendrimer using the formulas in Table 1.

\section{Results}

Theorem 1. Let $G$ be the PEG-cored PAMAM dendrimer. Then, the M-polynomial for $G$ is given as

$$
\begin{aligned}
M\left(C_{60}, x, y\right)= & 2 \times 4^{n} x y^{2}+4\left(4^{n}-1\right) x y^{3} \\
& +\left(12 \times 4^{n}+3 n-13\right) x^{2} y^{2} \\
& +\left(12 \times 4^{n}-6\right) x^{2} y^{3} .
\end{aligned}
$$

Proof. There are four types of edge partitions based on degrees of end vertices of each edge for the PEG-cored PAMAM dendrimer $G$ given in Figure 1 . The first edge partitions $E_{12}$ contains $2 \times 4^{n}$ edges, where $d_{u}=1$ and $d_{v}=2$. The second edge partition $E_{13}$ contains $4\left(4^{n}-1\right)$ edges, where $d_{u}=1$ and $d_{v}=3$. The third edge partition $E_{22}$ contains $\left(12 \times 4^{n}+3 n-13\right)$ edges, where $d_{u}=d_{v}=2$. The fourth edge partition $E_{23}$ contains $\left(12 \times 4^{n}-6\right)$ edges, where $d_{u}=2$ and $d_{v}=3$.

Therefore, the $M$-polynomial of $G$ is

$$
\begin{aligned}
M(G, x, y)= & \sum_{i \leq j} m_{i j} x^{i} y^{j}=2 \times 4^{n} x y^{2}+4\left(4^{n}-1\right) x y^{3} \\
& +\left(12 \times 4^{n}+3 n-13\right) x^{2} y^{2} \\
& +\left(12 \times 4^{n}-6\right) x^{2} y^{3} .
\end{aligned}
$$


TABle 1: The derived formulas to compute some degree-based topological indices from $M$-polynomial [18].

\begin{tabular}{lcc}
\hline Topological index & $\mathbf{f}(\mathbf{x}, \mathbf{y})$ & Derivation from $\mathbf{M}(\mathbf{G} ; \mathbf{x}, \mathbf{y})$ \\
\hline First Zagreb- $-M_{1}(G)$ & $x+y$ & $\left(D_{x}+D_{y}\right)(M(G ; x, y))_{x=y=1}$ \\
Second Zagreb- $-M_{2}(G)$ & $x y$ & $\left(D_{x} D_{y}\right)(M(G ; x, y))_{x=y=1}$ \\
Second modified Zagreb- $-{ }^{m} M_{2}(G)$ & $1 / x y$ & $\left(S_{x} S_{y}\right)(M(G ; x, y))_{x=y=1}$ \\
General Randić- $-R_{\alpha}(G), \alpha \in \mathbb{N}$ & $(x y)^{\alpha}$ & $\left(D_{x}^{\alpha} D_{y}^{\alpha}\right)(M(G ; x, y))_{x=y=1}$ \\
\hline
\end{tabular}

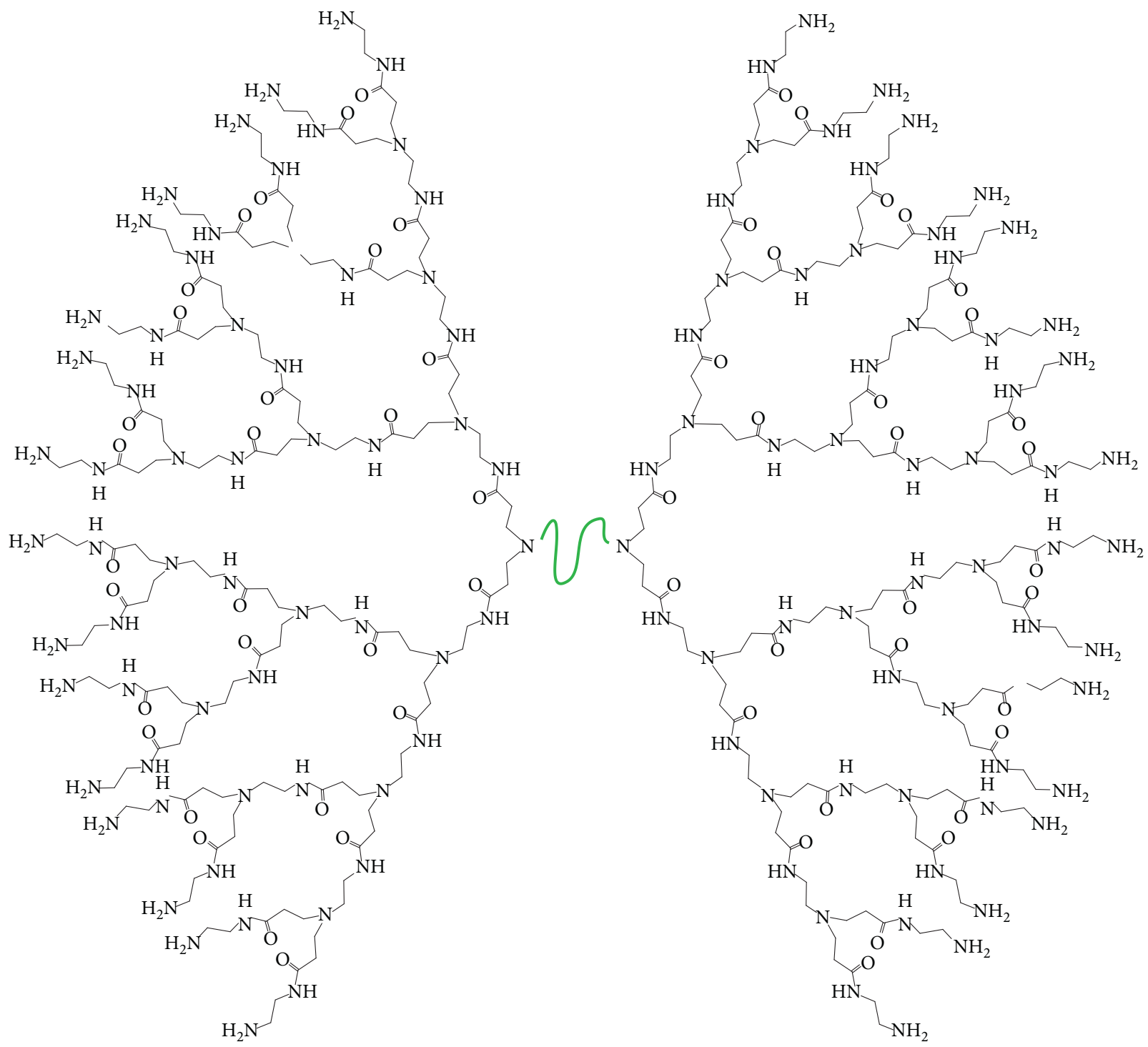

Figure 1: The structure of PEG-cored PAMAM dendrimer [16].

Proposition 1. The first Zagreb index $M_{1}(G)$, second Zagreb index $M_{2}(G)$, second modified Zagreb index ${ }^{m} M_{2}(G)$, and general Randic index $R_{\alpha}(G)$, where $\alpha \in \mathbb{N}$, for the PEG-cored PAMAM dendrimer $G$ are given as

(1) $M_{1}(G)=130 \times 4^{n}+12 n-98$

(2) $M_{2}(G)=136 \times 4^{n}+12 n-100$
(3) ${ }^{m} M_{2}(G)=(22 / 3) \times 4^{n}+(3 / 4) n-(67 / 4)$

(4) $R(G)=(\sqrt{2}+(4 / \sqrt{3})+(12 / \sqrt{6})+6) \times 4^{n}+$ $(3 / 2) n-((13 / 2)+(4 / \sqrt{3})+(6 / \sqrt{6}))$

Proof. Let $f(x, y)$ be the $M$-polynomial of $G$. Then, 


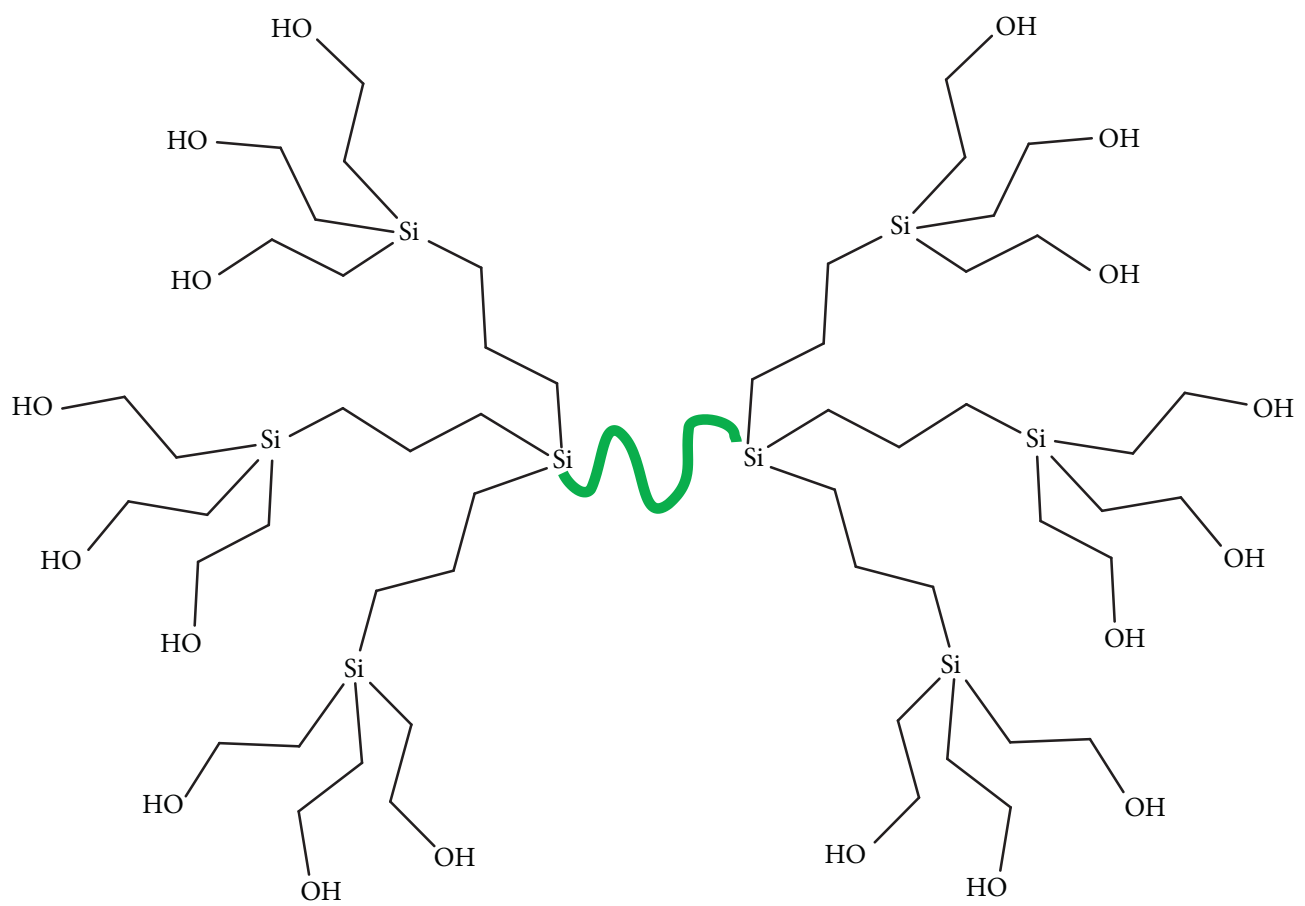

Figure 2: PEG-cored carbosilane dendrimer [16].

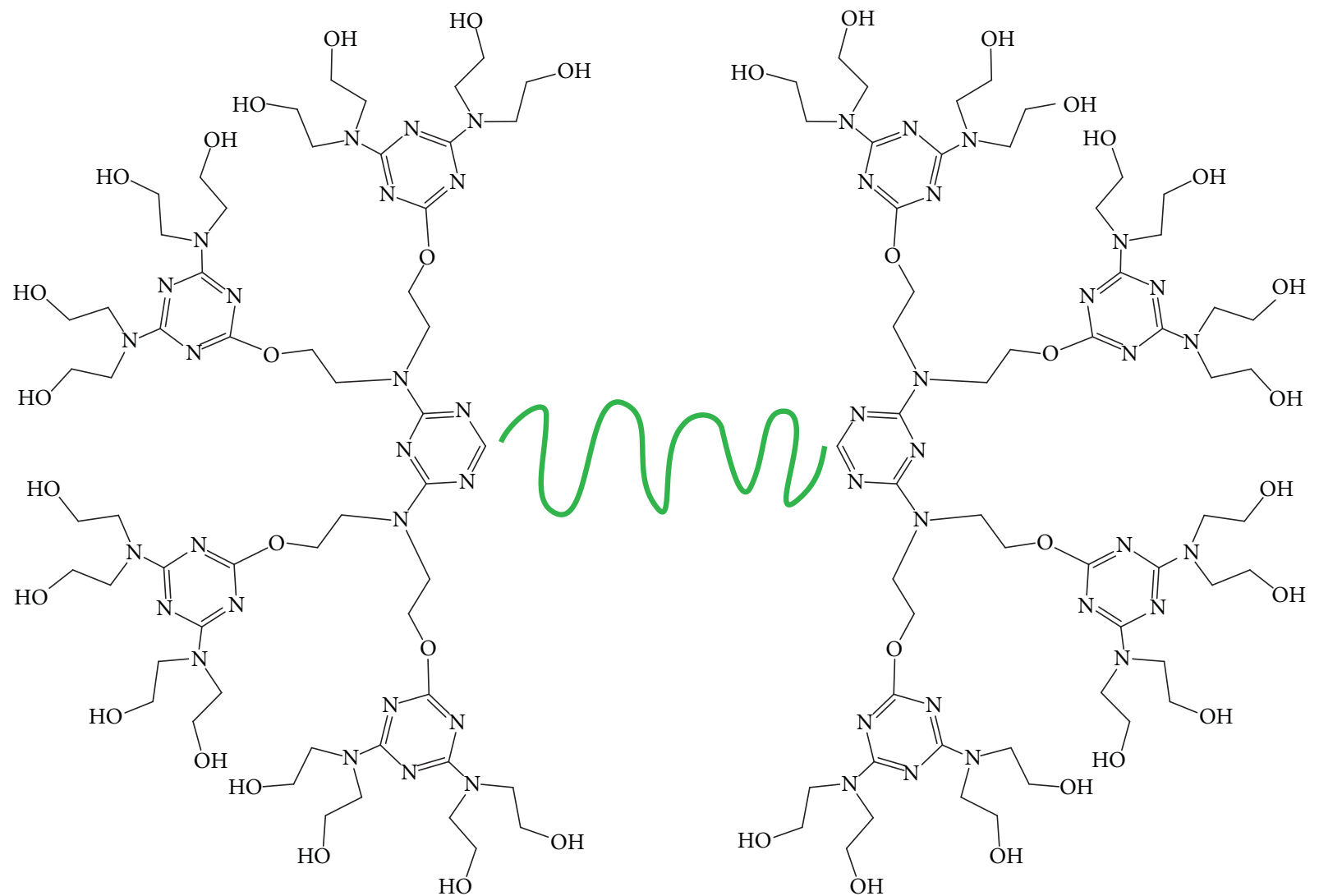

Figure 3: PEG-cored triazine dendrimer [16]. 


$$
\begin{aligned}
D_{x}(f(x, y)) & =2 \times 4^{n} x y^{2}+4\left(4^{n}-1\right) x y^{3}+2\left(12 \times 4^{n}+3 n-13\right) x^{2} y^{2}+2\left(12 \times 4^{n}-6\right) x^{2} y^{3}, \\
D_{y}(f(x, y)) & =4 \times 4^{n} x y^{2}+12\left(4^{n}-1\right) x y^{3}+2\left(12 \times 4^{n}+3 n-13\right) x^{2} y^{2}+3\left(12 \times 4^{n}-6\right) x^{2} y^{3}, \\
D_{x} D_{y}(f(x, y)) & =4 \times 4^{n} x y^{2}+12\left(4^{n}-1\right) x y^{3}+4\left(12 \times 4^{n}+3 n-13\right) x^{2} y^{2}+6\left(12 \times 4^{n}-6\right) x^{2} y^{3}, \\
S_{y}(f(x, y)) & =4^{n} x y^{2}+\frac{4}{3}\left(4^{n}-1\right) x y^{3}+\frac{1}{2}\left(12 \times 4^{n}+3 n-13\right) x^{2} y^{2}+\frac{1}{3}\left(12 \times 4^{n}-6\right) x^{2} y^{3}, \\
S_{x} S_{y}(f(x, y)) & =4^{n} x y^{2}+\frac{4}{3}\left(4^{n}-1\right) x y^{3}+\frac{1}{4}\left(12 \times 4^{n}+3 n-13\right) x^{2} y^{2}+\frac{1}{6}\left(12 \times 4^{n}-6\right) x^{2} y^{3}, \\
D_{y}^{\alpha}(f(x, y)) & =2^{\alpha+1} \times 4^{n} x y^{2}+4 \times 3^{\alpha}\left(4^{n}-1\right) x y^{3}+2^{\alpha} \times\left(12 \times 4^{n}+3 n-13\right) x^{2} y^{2}+3^{\alpha} \times\left(12 \times 4^{n}-6\right) x^{2} y^{3}, \\
D_{x}^{\alpha} D_{y}^{\alpha}(f(x, y)) & =2^{\alpha+1} \times 4^{n} x y^{2}+4 \times 3^{\alpha}\left(4^{n}-1\right) x y^{3}+4^{\alpha} \times\left(12 \times 4^{n}+3 n-13\right) x^{2} y^{2}+6^{\alpha} \times\left(12 \times 4^{n}-6\right) x^{2} y^{3} .
\end{aligned}
$$

Using the derived formulas of $M$-polynomial given in Table 1, we find

(1) $M_{1}(G)=\left.\left(D_{x}+D_{y}\right)(f(x, y))\right|_{x=y=1}=130 \times 4^{n}+$ $12 n-98$

(2) $M_{2}(G)=\left.D_{x} D_{y}(f(x, y))\right|_{x=y=1}=$ $136 \times 4^{n}+12 n-100$

(3) ${ }^{m} M_{2}(G)=\left.S_{x} S_{y}(f(x, y))\right|_{x=y=1}=(22 / 3) \times 4^{n}+$ $(3 / 4) n-(67 / 4)$

(4) $R(G)=\left.D_{x}^{\alpha} D_{y}^{\alpha}(f(x, y))\right|_{x=y=1, \alpha=-1 / 2}=(\sqrt{2}+$ $(4 / \sqrt{3})+(12 / \sqrt{6})+6) \times 4^{n}+$ $(3 / 2) n-((13 / 2)+(4 / \sqrt{3})+(6 / \sqrt{6}))$

Theorem 2. Let $G$ be the PEG-cored carbosilane dendrimer. Then, the M-polynomial for $G$ is given as

$$
\begin{aligned}
M\left(C_{60}, x, y\right)= & 6 \times 3^{n} x y^{2}+\left(11 \times 3^{n}+3 n-6\right) x^{2} y^{2} \\
& +\left(12 \times 3^{n}-4\right) x^{2} y^{4} .
\end{aligned}
$$

Proof. There are three types of edge partitions based on degrees of end vertices of each edge for the PEG-cored carbosilane dendrimer $G$ given in Figure 2. The first edge partitions $E_{12}$ contains $6 \times 3^{n}$ edges, where $d_{u}=1$ and $d_{v}=2$.
The second edge partition $E_{22}$ contains $\left(11 \times 3^{n}+3 n-6\right)$ edges, where $d_{u}=d_{v}=2$. The third edge partition $E_{24}$ contains $\left(12 \times 3^{n}-4\right)$ edges, where $d_{u}=2$ and $d_{v}=4$.

Therefore, the $M$-polynomial of $G$ is

$$
\begin{aligned}
M(G, x, y)= & \sum_{i \leq j} m_{i j} x^{i} y^{j}=6 \times 3^{n} x y^{2} \\
& +\left(11 \times 3^{n}+3 n-6\right) x^{2} y^{2} \\
& +\left(12 \times 3^{n}-4\right) x^{2} y^{4} .
\end{aligned}
$$

Proposition 2. The first Zagreb index $M_{1}(G)$, second Zagreb index $M_{2}(G)$, second modified Zagreb index ${ }^{m} M_{2}(G)$, and general Randić index $R_{\alpha}(G)$, where $\alpha \in \mathbb{N}$, for the PEG-cored carbosilane dendrimer $G$ are given as

(1) $M_{1}(G)=134 \times 3^{n}+12 n-48$

(2) $M_{2}(G)=152 \times 3^{n}+12 n-56$

(3) ${ }^{m} M_{2}(G)=(29 / 4) \times 3^{n}+(3 / 4) n-2$

(4) $R(G)=(6 \sqrt{2}+(11 / 2)) \times 3^{n}+(3 / 2) n-(3+\sqrt{2})$

Proof. Let $f(x, y)$ be the $M$-polynomial of $G$. Then,

$$
\begin{aligned}
D_{x}(f(x, y)) & =6 \times 3^{n} x y^{2}+2\left(11 \times 3^{n}+3 n-6\right) x^{2} y^{2}+2\left(12 \times 3^{n}-4\right) x^{2} y^{4}, \\
D_{y}(f(x, y)) & =12 \times 3^{n} x y^{2}+2\left(11 \times 3^{n}+3 n-6\right) x^{2} y^{2}+4\left(12 \times 3^{n}-4\right) x^{2} y^{4}, \\
D_{x} D_{y}(f(x, y)) & =12 \times 3^{n} x y^{2}+4\left(11 \times 3^{n}+3 n-6\right) x^{2} y^{2}+8\left(12 \times 3^{n}-4\right) x^{2} y^{4}, \\
S_{y}(f(x, y)) & =3 \times 3^{n} x y^{2}+\frac{\left(11 \times 3^{n}+3 n-6\right)}{2} x^{2} y^{2}+\left(3 \times 3^{n}-1\right) x^{2} y^{4}, \\
S_{x} S_{y}(f(x, y)) & =3 \times 3^{n} x y^{2}+\frac{\left(11 \times 3^{n}+3 n-6\right)}{4} x^{2} y^{2}+\frac{\left(3 \times 3^{n}-1\right)}{2} x^{2} y^{4}, \\
D_{y}^{\alpha}(f(x, y)) & =6 \times 2^{\alpha} \times 3^{n} x y^{2}+2^{\alpha} \times\left(11 \times 3^{n}+3 n-6\right) x^{2} y^{2}+4^{\alpha} \times\left(12 \times 3^{n}-4\right) x^{2} y^{4}, \\
D_{x}^{\alpha} D_{y}^{\alpha}(f(x, y)) & =6 \times 2^{\alpha} \times 3^{n} x y^{2}+4^{\alpha} \times\left(11 \times 3^{n}+3 n-6\right) x^{2} y^{2}+8^{\alpha} \times\left(12 \times 3^{n}-4\right) x^{2} y^{4} .
\end{aligned}
$$


Using the derived formulas of $M$-polynomial given in Table 1, we find

(1) $M_{1}(G)=\left.\left(D_{x}+D_{y}\right)(f(x, y))\right|_{x=y=1}=134 \times 3^{n}+$ $12 n-48$

(2) $M_{2}(G)=\left.D_{x} D_{y}(f(x, y))\right|_{x=y=1}=$ $152 \times 3^{n}+12 n-56$

(3) ${ }^{m} M_{2}(G)=\left.S_{x} S_{y}(f(x, y))\right|_{x=y=1}=(29 / 4) \times 3^{n}+$ $(3 / 4) n-2$

(4) $R(G)=\left.D_{x}^{\alpha} D_{y}^{\alpha}(f(x, y))\right|_{x=y=1,} \alpha=-1 / 2=(6 \sqrt{2}+$ $(11 / 2)) \times 3^{n}+(3 / 2) n-(3+\sqrt{2})$

Theorem 3. Let $G$ be the PEG-cored triazine dendrimer. Then, the M-polynomial for $G$ is given as

$$
\begin{aligned}
M\left(C_{60}, x, y\right)= & 6 \times 3^{n} x y^{2}+\left(11 \times 3^{n}+3 n-6\right) x^{2} y^{2} \\
& +\left(12 \times 3^{n}-4\right) x^{2} y^{4} .
\end{aligned}
$$

Proof. There are three types of edge partitions based on degrees of end vertices of each edge for the PEG-cored triazine dendrimer. The first edge partitions $E_{12}$ contains $8 \times 4^{n}$ edges, where $d_{u}=1$ and $d_{v}=2$. The second edge partition $E_{22}$ contains $1 / 3\left(40 \times 4^{n}+9 n-19\right)$ edges, where $d_{u}=d_{v}=2$. The third edge partition $E_{23}$ contains $\left(24 \times 4^{n}-\right.$ 6) edges, where $d_{u}=2$ and $d_{v}=3$.

Therefore, the $M$-polynomial of $G$ is

$$
\begin{aligned}
M(G, x, y)= & \sum_{i \leq j} m_{i j} x^{i} y^{j}=8 \times 4^{n} x y^{2} \\
& +\frac{1}{3}\left(40 \times 4^{n}+9 n-19\right) x^{2} y^{2} \\
& +\left(24 \times 4^{n}-6\right) x^{2} y^{3} .
\end{aligned}
$$

Proposition 3. The first Zagreb index $M_{1}(G)$, the second Zagreb index $M_{2}(G)$, second modified Zagreb index ${ }^{m} M_{2}(G)$, and the general Randic index $R_{\alpha}(G)$, where $\alpha \in \mathbb{N}$, for the PEG-cored triazine dendrimer $G$ are given as

(1) $M_{1}(G)=(529 / 3) \times 4^{n}+12 n-(166 / 3)$

(2) $M_{2}(G)=(640 / 3) \times 4^{n}+12 n-(184 / 3)$

(3) ${ }^{m} M_{2}(G)=(34 / 3) \times 4^{n}+(3 / 4) n-(31 / 12)$

(4) $R(G)=((8 / \sqrt{2})+(24 / \sqrt{6})+(20 / 3)) \times 4^{n}+$

$(3 / 2) n-((19 / 6)+(6 / \sqrt{6}))$

Proof. Let $f(x, y)$ be the $M$-polynomial of $G$. Then,

$$
\begin{aligned}
D_{x}(f(x, y)) & =8 \times 4^{n} x y^{2}+\frac{2}{3}\left(40 \times 4^{n}+9 n-19\right) x^{2} y^{2}+2\left(24 \times 4^{n}-6\right) x^{2} y^{3}, \\
D_{y}(f(x, y)) & =16 \times 4^{n} x y^{2}+\frac{2}{3}\left(40 \times 4^{n}+9 n-19\right) x^{2} y^{2}+3\left(24 \times 4^{n}-6\right) x^{2} y^{3}, \\
D_{x} D_{y}(f(x, y)) & =16 \times 4^{n} x y^{2}+\frac{4}{3}\left(40 \times 4^{n}+9 n-19\right) x^{2} y^{2}+6\left(24 \times 4^{n}-6\right) x^{2} y^{3}, \\
S_{y}(f(x, y)) & =4 \times 4^{n} x y^{2}+\frac{1}{6}\left(40 \times 4^{n}+9 n-19\right) x^{2} y^{2}+\left(8 \times 4^{n}-2\right) x^{2} y^{3}, \\
S_{x} S_{y}(f(x, y)) & =4 \times 4^{n} x y^{2}+\frac{1}{12}\left(40 \times 4^{n}+9 n-19\right) x^{2} y^{2}+\left(4 \times 4^{n}-1\right) x^{2} y^{3}, \\
D_{y}^{\alpha}(f(x, y)) & =8 \times 2^{\alpha} \times 4^{n} x y^{2}+\frac{2^{\alpha}}{3}\left(40 \times 4^{n}+9 n-19\right) x^{2} y^{2}+3^{\alpha}\left(24 \times 4^{n}-6\right) x^{2} y^{3}, \\
D_{x}^{\alpha} D_{y}^{\alpha}(f(x, y)) & =8 \times 2^{\alpha} \times 4^{n} x y^{2}+\frac{4^{\alpha}}{3}\left(40 \times 4^{n}+9 n-19\right) x^{2} y^{2}+6^{\alpha}\left(24 \times 4^{n}-6\right) x^{2} y^{3} .
\end{aligned}
$$

Using the derived formulas of $M$-polynomial given in Table 1, we find

$$
\begin{aligned}
& \text { (1) } M_{1}(G)=\left.\left(D_{x}+D_{y}\right)(f(x, y))\right|_{x=y=1}=(529 / 3) \times \\
& 4^{n}+12 n-(166 / 3) \\
& \text { (2) } M_{2}(G)=\left.D_{x} D_{y}(f(x, y))\right|_{x=y=1}=(640 / 3) \times 4^{n}+ \\
& 12 n-(184 / 3) \\
& \text { (3) }{ }^{m} M_{2}(G)=\left.S_{x} S_{y}(f(x, y))\right|_{x=y=1}=(34 / 3) \times 4^{n}+
\end{aligned}
$$
$(3 / 4) n-(31 / 12)$

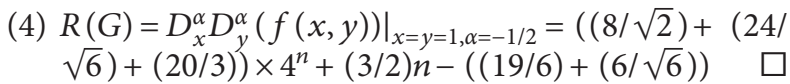

\section{Conclusion}

Chemical graph theory plays an important role in studying the molecular structure of the chemical compounds. Calculating the topological indices of the chemical structure using graph theory is important to study the large amount of 
information about their physical properties. In this study, we derive the $M$-polynomials for the PEG-cored PAMAM, carbosilane, and poly (lysine) dendrimers and calculate their first, second, and second modified Zagreb indices and the Randić index. This work may provide the role in determining the properties of these dendrimeric structures.

\section{Data Availability}

The data used to support the findings of this study are included within the article.

\section{Conflicts of Interest}

The authors declare that they have no conflicts of interest.

\section{References}

[1] E. Estrada, G. Patlewicz, and E. Uriarte, "From molecular graphs to drugs. A review on the use of topological indices in drug design and discovery," Indian Journal of Chemistry Section A, vol. 42, pp. 1315-1329, 2003.

[2] E. Estrada and E. Uriarte, "Recent advances on the role of topological indices in drug discovery research," Current Medicinal Chemistry, vol. 8, no. 13, pp. 1573-1588, 2001.

[3] R. Zanni, M. Galvez-Llompart, R. García-Domenech, and J. Galvez, "Latest advances in molecular topology applications for drug discovery," Expert Opinion on Drug Discovery, vol. 10, no. 9, pp. 945-957, 2015.

[4] D. H. Rouvray, "The Search for Useful Topological Indices in Chemistry: topological indices promise to have far-reaching applications in fields as diverse as bonding theory, cancer research, and drug design," American Scientist, vol. 61, pp. 729-735, 1973.

[5] J. Gálvez, M. Gálvez-Llompart, and R. García-Domenech, "Molecular topology as a novel approach for drug discovery," Expert Opinion on Drug Discovery, vol. 7, no. 2, pp. 133-153, 2012.

[6] N. De, "Computing reformulated first Zagreb index of some chemical graphs as an application of generalized hierarchical product of graphs," Open Journal of Mathematical Sciences, vol. 2, no. 1, pp. 338-350, 2018.

[7] M. Riaz, W. Gao, A. Q. Baig, W. Gao, and A. Qudair Baig, " $M$ polynomials and degree-based topological indices of some families of convex polytopes," Open Journal of Mathematical Sciences, vol. 2, no. 1, pp. 18-28, 2018.

[8] M. S. Sardar, S. Zafar, S. Zafar, and M. R. Farahani, "The generalized Zagreb index of capra-designed planar benzenoid series \$Ca_k(C_6)\$," Open Journal of Mathematical Sciences, vol. 1, no. 1, pp. 44-51, 2017.

[9] N. De, "Hyper Zagreb index of bridge and chain graphs," Open Journal of Mathematical Sciences, vol. 2, pp. 1-17, 2018.

[10] T. P. Jude, E. Panchadcharam, and K. Masilamani, "Computation of Zagreb and Randić indices of two biodegradable dendrimers used in cancer therapy," Ceylon Journal of Science, vol. 48, no. 4, pp. 359-366, 2019.

[11] S. M. Kang, M. A. Zahid, A. u. R. Virk, W. Nazeer, and W. Gao, "Calculating the degree-based topological indices of dendrimers," Open Chemistry, vol. 16, no. 1, pp. 681-688, 2018.

[12] A. Aslam, Y. Bashir, S. Ahmad, and W. Gao, "On topological indices of certain dendrimer structures," Zeitschrift für Naturforschung A, vol. 72, no. 6, pp. 559-566, 2017.
[13] J. Zhu and X. Shi, "Dendrimer-based nanodevices for targeted drug delivery applications," Journal of Materials Chemistry B, vol. 1, no. 34, pp. 4199-4211, 2013.

[14] D. A. Tomalia, "Birth of a new macromolecular architecture: dendrimers as quantized building blocks for nanoscale synthetic polymer chemistry," Progress in Polymer Science, vol. 30, no. 3-4, pp. 294-324, 2005.

[15] M. Kalomiraki, K. Thermos, and N. A. Chaniotakis, "Dendrimers as tunable vectors of drug delivery systems and biomedical and ocular applications," International Journal of Nanomedicine, vol. 11, pp. 1-12, 2016.

[16] Y. Cheng, L. Zhao, Y. Li, and T. Xu, "Design of biocompatible dendrimers for cancer diagnosis and therapy: current status and future perspectives," Chemical Society Reviews, vol. 40, no. 5, pp. 2673-2703, 2011.

[17] H. Wiener, "Structural determination of paraffin boiling points," Journal of the American Chemical Society, vol. 69, no. 1, pp. 17-20, 1947.

[18] I. Gutman and N. Trinajstić, "Graph theory and molecular orbitals. Total $\varphi$-electron energy of alternant hydrocarbons," Chemical Physics Letters, vol. 17, no. 4, pp. 535-538, 1972.

[19] J. Hao, "Theorems about Zagreb indices and modified Zagreb indices," MATCH Communications in Mathematical and in Computer Chemistry, vol. 65, pp. 659-670, 2011.

[20] M. Randić, "On Characterization of molecular branching," Journal of the American Chemical Society, vol. 97, pp. 66096615, 1974.

[21] X. Li and Y. Shi, "A survey on the Randić index," MATCH Communications in Mathematical and in Computer Chemistry, vol. 59, pp. 127-156, 2008.

[22] E. Deutsch and S. Klavzar, "M-polynomial and degree-based topological indices, Iran," Journal of Mathematical Chemistry, vol. 6, pp. 93-102, 2015. 